\title{
BMJ Open Quality Optimisation of diagnosis and treatment of heart failure in a primary care setting
}

To cite: Bakhai S, Bhardwaj A, Phan $\mathrm{H}$, et al. Optimisation of diagnosis and treatment of heart failure in a primary care setting. BMJ Open Quality 2019;8:e000660. doi:10.1136/ bmjoq-2019-000660

Received 14 February 2019 Revised 6 September 2019 Accepted 30 September 2019

Check for updates

(C) Author(s) (or their employer(s)) 2019. Re-use permitted under CC BY-NC. No commercial re-use. See rights and permissions. Published by BMJ.

${ }^{1}$ Medicine, Division of Internal Medicine, University at Buffalo-The State University of New York, Buffalo, New York, USA

${ }^{2}$ Medicine, Division of Behavioral Medicine, University at Buffalo-The State University of New York, Buffalo, New York, USA

${ }^{3}$ Medicine, Divison of Allergy, Immunology \& Rheumatology, University at Buffalo-The State University of New York, Buffalo, New York, USA

Correspondence to

Dr Smita Bakhai;

sybakhai@buffalo.edu

\author{
Smita Bakhai, ${ }^{1}$ Aishwarya Bhardwaj, ${ }^{1}$ Huy Phan, ${ }^{1}$ Shane Varghese,${ }^{1}$ \\ Gregory D Gudleski, ${ }^{2}$ Jessica L Reynolds ${ }^{3}$
}

\section{ABSTRACT}

Background Heart failure (HF) is one of the leading causes of emergency department visits and hospital admissions in the USA. We identified a gap in the diagnosis and the use of guideline-directed medical therapy in patients with $\mathrm{HF}$ at the internal medicine clinic.

Aim To improve the diagnosis and treatment of HF, as well as to reduce emergency department visits and hospitalisation over 12 months in patients aged $40-75$ years.

Methods The multidisciplinary quality improvement (QI) team performed a root cause analysis and identified barriers to optimal guideline-directed medical therapy. Rates of patients on guideline-directed medical therapy with systolic HF diagnosis, emergency department visits and hospital admissions were the outcome measures. The process measures included echocardiogram order and completion rates, and rates of accurate classification of $\mathrm{HF}$ from the baseline rate of less than $10 \%$. We used the focus, analyse, develop, execute and evaluate (FADE) model with five improvement cycles. The major components of interventions included (1) leveraging health information technology; (2) optimising teamwork; and (3) providing education to patients, physicians and internal medicine clinic staff. Data were analysed using statistical process control and run charts.

Results We observed a reduction in the total number of emergency department visits (160 vs 108), hospital admissions (117 vs 114) and observation visits (22 vs 16) comparing the 1-year preproject and 1-year postproject periods. An increase in the use of ACE inhibitors or angiotensin receptor blockers occurred from the baseline rate of $20 \%-37 \%$ during the second half of the project and was sustained at $71.4 \%$ (median) during 6 months of the postproject period.

Conclusions We achieved a sustainable increase in the accurate diagnosis of $\mathrm{HF}$ and achieved $80 \%$ diagnosis during the 6-month poststudy period.

\section{INTRODUCTION}

Heart failure (HF), a chronic, progressive condition, occurs when the heart muscle is unable to pump enough blood to meet the body's demands for blood and oxygen. ${ }^{1} \mathrm{HF}$ results from a structural or functional impairment of ventricular filling or ejection of blood. ${ }^{2}$ In the USA, approximately 5.7 million adults are living with $\mathrm{HF}^{1-3}$ and occurrence rates increase with age. ${ }^{23} \mathrm{HF}$ is a growing healthcare burden with 2012 cost estimates around $\$ 30.7$ billion. ${ }^{4}$ $\mathrm{HF}$ is a leading diagnosis for hospitalisation and hospital readmissions. ${ }^{1-3}$ From 2009 to 2012, the median risk-standardised 30-day readmission rate for $\mathrm{HF}$ was $23.0 \% .^{5}$ The overall implications of HF include a negative impact on patients' quality of life, increased morbidity and mortality, and increased number of avoidable hospitalisations. ${ }^{6-8}$

$\mathrm{HF}$ with reduced ejection fraction is defined as a left ventricular ejection fraction of less than 40\%. The 2013 American College of Cardiology Foundation/American Heart Association Task Force guidelines for $\mathrm{HF}$ management include the use of ACE inhibitors or angiotensin receptor blockers (ARBs), in conjunction with evidence-based beta blockers and aldosterone antagonists for patients with reduced ejection fraction. ${ }^{2}$ The 2017 focused update of HF guidelines recommends the use of an ARBs with an angiotensin receptor-neprilysin inhibitor for the treatment of patients with $\mathrm{HF}$ with reduced ejection fraction. ${ }^{3}$ As HF is one of the leading causes of emergency department visits and hospitalisation readmissions, diagnosing and managing $\mathrm{HF}$ based on guideline-directed medical therapy have become a priority. ${ }^{1-3}$

The Affordable Care Act (2010) established the Hospital Readmissions Reduction Program, which is a value-based purchasing programme that reduces payments to hospitals with excess readmissions, including readmissions for HF. ${ }^{910}$ Furthermore, in 2014, New York State implemented the Delivery System Reform Incentive Payment programme. ${ }^{11}$ This incentive-based programme mandates that participating healthcare organisations reduce avoidable hospitalisations that include inappropriate admissions, readmissions and preventable emergency department use. ${ }^{11} 12$ The leadership at Erie County Medical Center, a safety-net hospital in Western New York, participated in both of these initiatives and was supportive of this quality improvement (QI) project. Roughly $15 \%$ of the patient population that uses the internal medicine clinic has a diagnosis of HF. We identified a gap in the accurate diagnosis and guideline-directed medical therapy 
for patients with HF. The aims of this QI project were to increase accurate classification of HF based on ejection fraction from the baseline rate of less than $10 \%$ to a rate of $30 \%$ and to increase the use of guideline-directed medical therapy within 12 months in patients aged 40-75 years with a diagnosis of systolic $\mathrm{HF}$ at the internal medicine clinic. The ultimate goal was to reduce emergency department visits and hospitalisations in patients with HF.

\section{METHODS}

\section{Setting}

We conducted a QI project in an academic internal medicine clinic located within a tertiary care safety-net hospital, Erie County Medical Center. The internal medicine clinic patient population consists of mostly urban, underprivileged and African-Americans $(68.42 \%)$. Patients use the internal medicine clinic as a longitudinal primary care clinic; the internal medicine clinic has an average of 700 monthly visits. The internal medicine clinic comprises a multidisciplinary care team including 35 residents from the University at Buffalo's Internal Medicine Residency Programme and four attending physicians.

\section{Design}

The QI team included a physician champion, nursing and ancillary staff, residents, attending physicians, a social worker, patients, and administrative and information technology staff. The QI team used specific, measurable, attainable, relevant and timely (SMART) objectives to define our aims. ${ }^{13}$ We used the six aims of changing the healthcare system, the safe, timely, effective, efficient, equitable and patient-centred (STEEEP) model for programme management (table 1). ${ }^{14}$ We designed this QI based on the focus, analyse, develop, execute and evaluate (FADE) QI model. ${ }^{16-18}$ We used SQUIRE V.2.0 guidelines for reporting this QI project. ${ }^{19}$ The QI team performed a root cause analysis using a fishbone diagram and identified the materials/methods, physician-related and patient-related barriers to optimal guideline-directed medical therapy of patients with HF (figure 1). We developed a process flow map to optimise opportunity to improve HF management (figure 2). In collaboration with the information technology department, we created an electronic patient database from various electronic health records (EHRs) as Erie County Medical Center has different EHRs for inpatient and outpatient settings. Patient demographics, echocardiogram order date, echocardiogram completion date, current medications, past medications, specific classifications of HF (systolic HF, HF with normal ejection fraction or combined HF) based on the International Classification of Diseases, 10th Revision, diagnosis codes and emergency department visits, observation and hospitalisations were included in the registry. The purpose of this patient registry was to address care gaps, to review electronic reports of resource use for emergency department visits, observations and hospitalisations, and to generate electronic reports of echocardiogram order and echocardiogram completion rates. The physician champion and resident team leaders conducted multiple reviews for quality assurance and collaborated with information technology staff to ensure accuracy of the database. Furthermore, the QI team, in collaboration with the information technology staff, designed and implemented an electronic tracking system for Erie County Medical Center hospital discharges and emergency department visits to facilitate scheduling of timely clinic follow-up appointments for internal medicine clinic patients.

The baseline rate for accurate classification of patients with HF was about $10 \%$; the use of ACE or ARBs was 20\%; and there was no use of spironolactone in patients with systolic HF. All patients with HF diagnosis were on a beta blocker. The baseline rate was obtained by retrospective review of the database of eligible patients seen at least

\section{Table 1 Institute of Medicine's six-aim STEEEP model}

\begin{tabular}{ll}
\hline Safe & $\begin{array}{l}\text { Guideline-directed medical therapy has been proven to have greater benefit to overall mortality and } \\
\text { quality of life in patients with HF in comparison to risks of adverse effects } \\
\text { Identification of eligible patients due for echocardiogram and initial medication reconciliation performed } \\
\text { during physicians' administrative task time; and recheck at the time of the clinic visit leading to timely } \\
\text { completion of the patient visit; monthly tracking of echocardiogram completion and HF classification } \\
\text { rates }\end{array}$ \\
Effective & $\begin{array}{l}\text { Guideline-directed medical therapy for systolic HF has been proven to improve ejection fraction and } \\
\text { mortality. }\end{array}$ \\
Efficient & $\begin{array}{l}\text { Prescribing guideline-directed medical therapy for patients with HF may lead to reduction in emergency } \\
\text { department visits and hospital readmission rates. }\end{array}$ \\
Equitable & $\begin{array}{l}\text { Intent to provide guideline-directed medical therapy to all patients and to provide certain medication } \\
\text { classes over others based on ethnicity, such as use of ACE or angiotensin receptor blockers in } \\
\text { Caucasians, and hydralazine and nitrates in African-Americans }\end{array}$ \\
Patient-centred & $\begin{array}{l}\text { Ultimate aim is to improve patient mortality and quality of life with patient engagement and shared } \\
\text { decision making }\end{array}$
\end{tabular}

HF, heart failure; STEEEP, safe, timely, effective, efficient, equitable and patient-centred. 


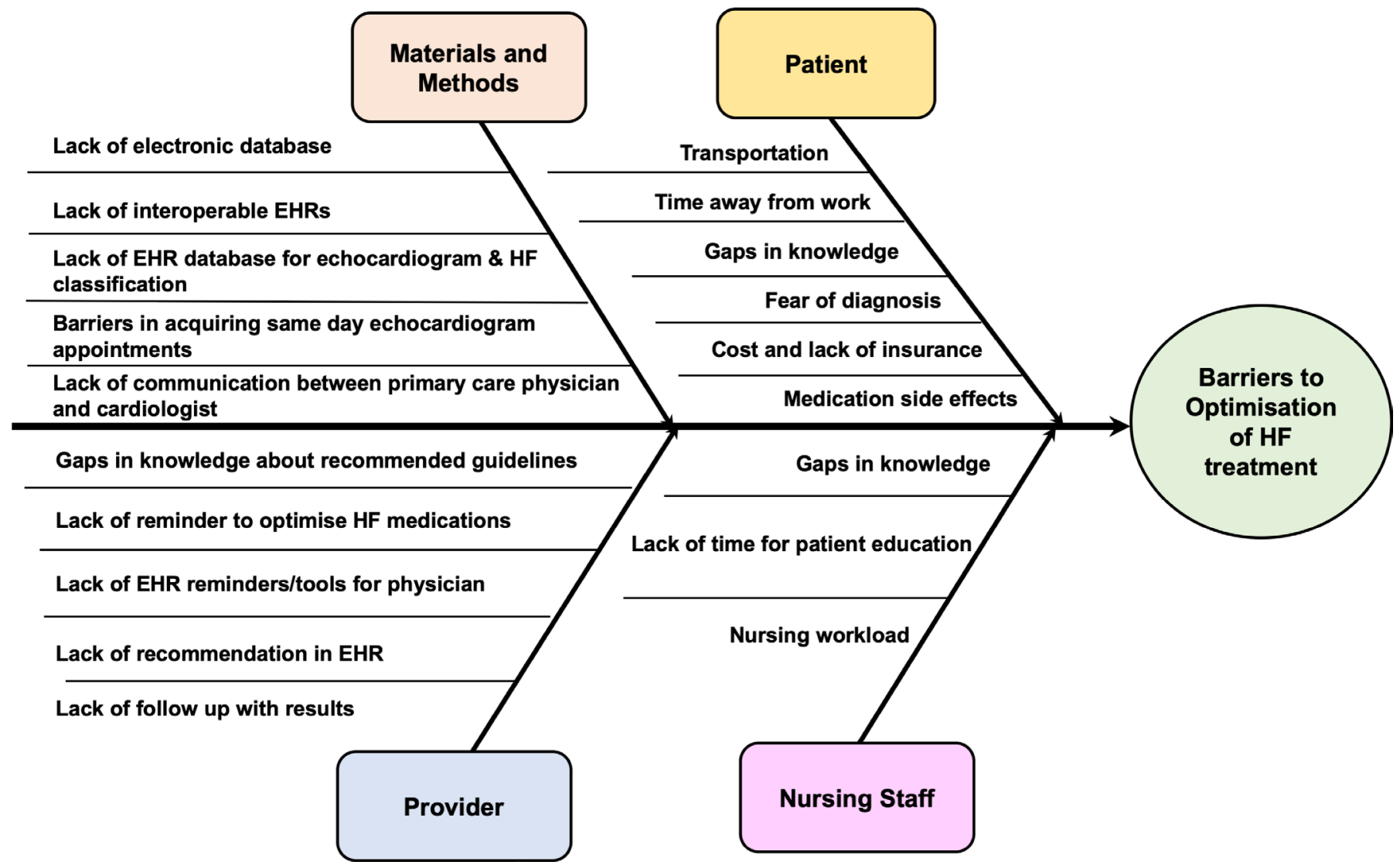

Figure 1 Fishbone diaphragm: root cause analysis identifying barriers to optimisation of HF treatment. HF, heart failure; EHR, electronic health record.

once within the past 12 months in the internal medicine clinic. We included male and female patients between the ages of 40 and 75 years, with a diagnosis of HF, leg oedema or shortness of breath, evaluated in the clinic from July 2016 to June 2017. Patients were eligible for an echocardiogram if they had no prior echocardiogram or the echocardiogram was completed more than 2 years ago. Physicians identified eligible patients for echocardiogram by reviewing the patients' EHR. During subsequent follow-up visits, the physicians reviewed the barriers to echocardiogram after the patients missed an appointment and referred the patients for echocardiogram.

\section{Measurements}

The outcome measures included (1) rates of patients on guideline-directed medical therapy with systolic HF diagnosis: (a) ACE inhibitors to $30 \%$ from the baseline rate of 20\%, (b) spironolactone and (c) sustaining the use of beta blockers in all patients with HF; and (2) emergency department visits and hospital admissions. The process measures included echocardiogram order and completion rates and accurate classification of $\mathrm{HF}$ from the baseline rate of less than $10 \%-30 \%$ based on the ejection fraction of heart function. The balance measures included (1) an increase in patient wait time during the internal medicine clinic visit due to extra time needed to provide education on diagnosis and medications to patients, (2) poor access to echocardiogram due to increase in demand and (3) access to medications due to cost and ability of patients to obtain their prescriptions. The main focus of this QI was to improve the diagnosis and treatment of HF specifically through the use of echocardiogram. An echocardiogram is required to assess cardiac function and ejection fraction is used to classify systolic HF and to exclude other diagnoses. ${ }^{23}$ Therefore, echocardiogram order and completions rates and accurate HF diagnosis were selected as the main process measures. Once $\mathrm{HF}$ is classified accurately, guideline-directed medical therapy is crucial to reduce morbidity and mortality. ${ }^{23} \mathrm{We}$ selected guideline-directed medical therapy and rates of emergency department visits and hospital admissions as the outcome measures.

\section{Strategy}

Improvement cycle 1 (July-September 2016): physician and staff training

Resident team leaders administered a pretest to identify gaps in the residents' knowledge of HF guidelines. We educated residents and clinic staff through PowerPoint presentations in a small group discussion setting. Presentation topics included current HF guidelines and EHR workflow integration of ordering echocardiogram and prescriptions for guideline-directed medical therapy.

Improvement cycle 2 (October-December 2016): creation of EHR database and education

The physician champion of this QI project collaborated with information technology staff and created an 


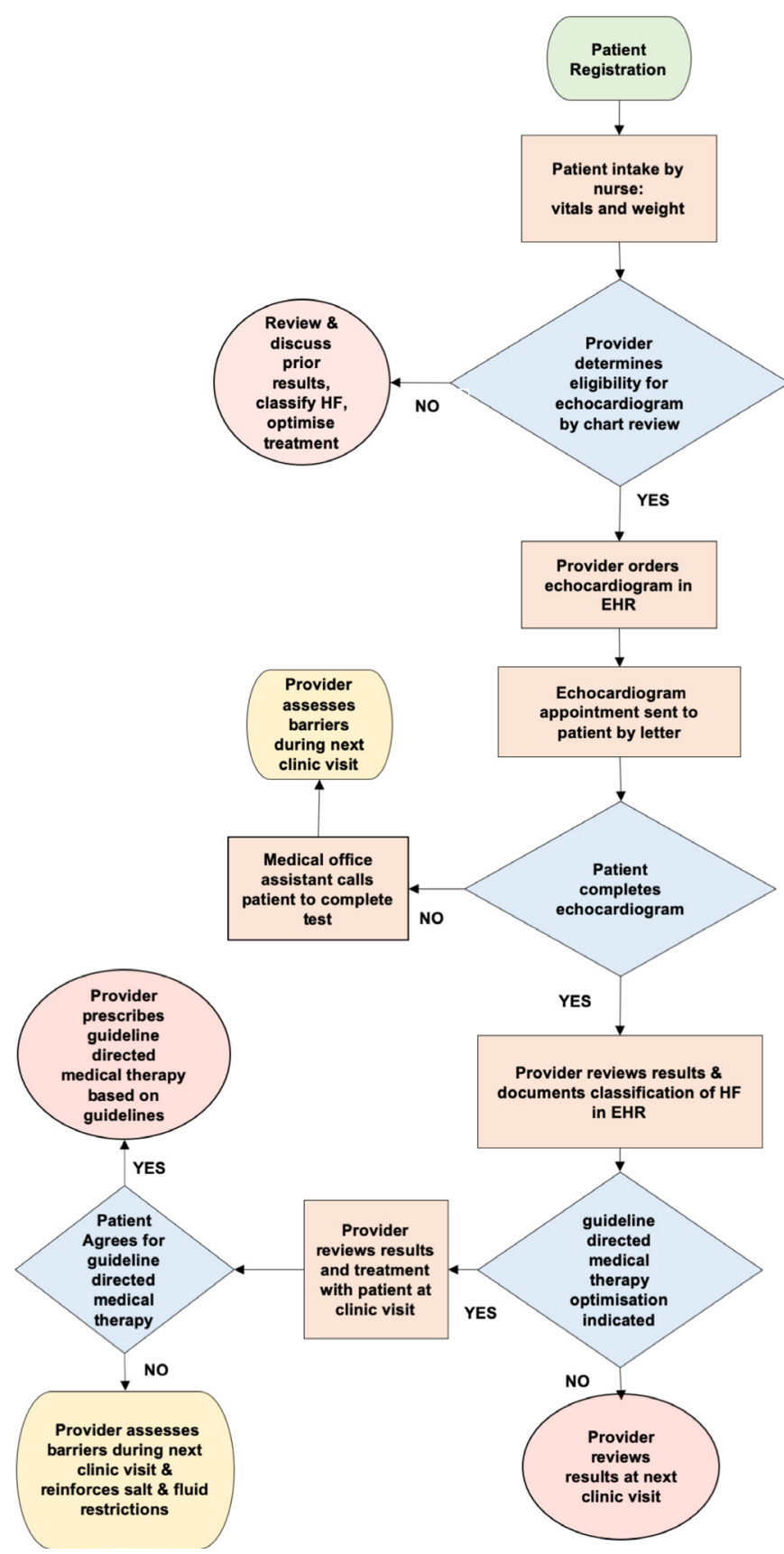

Figure 2 Process flow map. HF, heart failure; EHR, electronic health record.

electronic database of patients with HF. The team leaders provided training to the residents and internal medicine clinic physicians regarding the use of the database for this QI project.

\section{Improvement cycle 3 (January-March 2017): physician reminder}

Resident team leaders created and placed pamphlets in residents' task boxes to remind them about classification and guideline-directed medical therapy for patients with HF (figure 3A). A clinical decision support tool for physicians was not built in the EHR. The physician champion discussed the need to build or purchase clinical decision support tools with information technology leadership; however, the cost was identified as the major barrier to the implementation of a clinical decision support tool. Therefore, residents developed pamphlets to remind physicians. Physicians discussed the results of the echocardiogram and the need for further guideline-directed medical therapy with patients, and offered generic medications based on patients' symptoms and HF classification. Physicians prescribed guideline-directed medical therapy after patients agreed to take the new medications. This patient-centered decision was made during the clinic visit with the patients to optimise guideline-directed medical therapy. Physicians also addressed barriers to patients' access (the cost and the ability of patients to obtain their prescriptions). Physicians referred patients to a social worker when they were concerned about the cost of medications.

Improvement cycle 4 (April-May 2017): stakeholder engagement The QI team continued to share progress with the internal medicine clinic staff and residents. Erie County Medical Center faced an EHR outage due to ransomware for 2 months. Paper medical records were implemented for documentation of clinic visit during the EHR outage. Physicians did not have access to previous medical records for about 1 week. Physicians were unable to verify if patients were eligible for echocardiogram. Echocardiogram order rates were affected during this time due to unavailability of previous medical records from the EHR. However, after a few weeks of outage, physicians had view access to the EHR and were able to order an echocardiogram for eligible patients using paper records. Echocardiograms were completed during the EHR outage; however, completed echocardiogram results were not automatically uploaded in the EHR and registry of patients with HF. The registry of patients with HF was based on completed echocardiogram results interfaced in the EHR. Erie County Medical Center had limited resources to enter the data manually.

\section{Improvement cycle 5 (June-2017):patient engagement and nursing education}

In order to create patient awareness of HF, resident team leaders designed patient education pamphlets and placed these materials in the examination rooms (figure 3B). Patients were actively engaged in this QI project. Team leaders created simplified instructions on the patient education pamphlet, designed with patients' feedback. Physicians and nursing staff educated patients regarding weight monitoring at home and dispensed weighing scales at no cost to patients. The nursing staff and physicians instructed patients on fluid management, and patients were instructed to notify physicians if they gained significant weight and to keep a diary of daily weight. Physicians also discussed increasing diuretics based on weight gain in selected patients.

\section{Data analysis}

Data analysis was performed using a weekly statistical process control chart and monthly run charts. We also 
Figure 3a. Physician Reminder Pamphlets

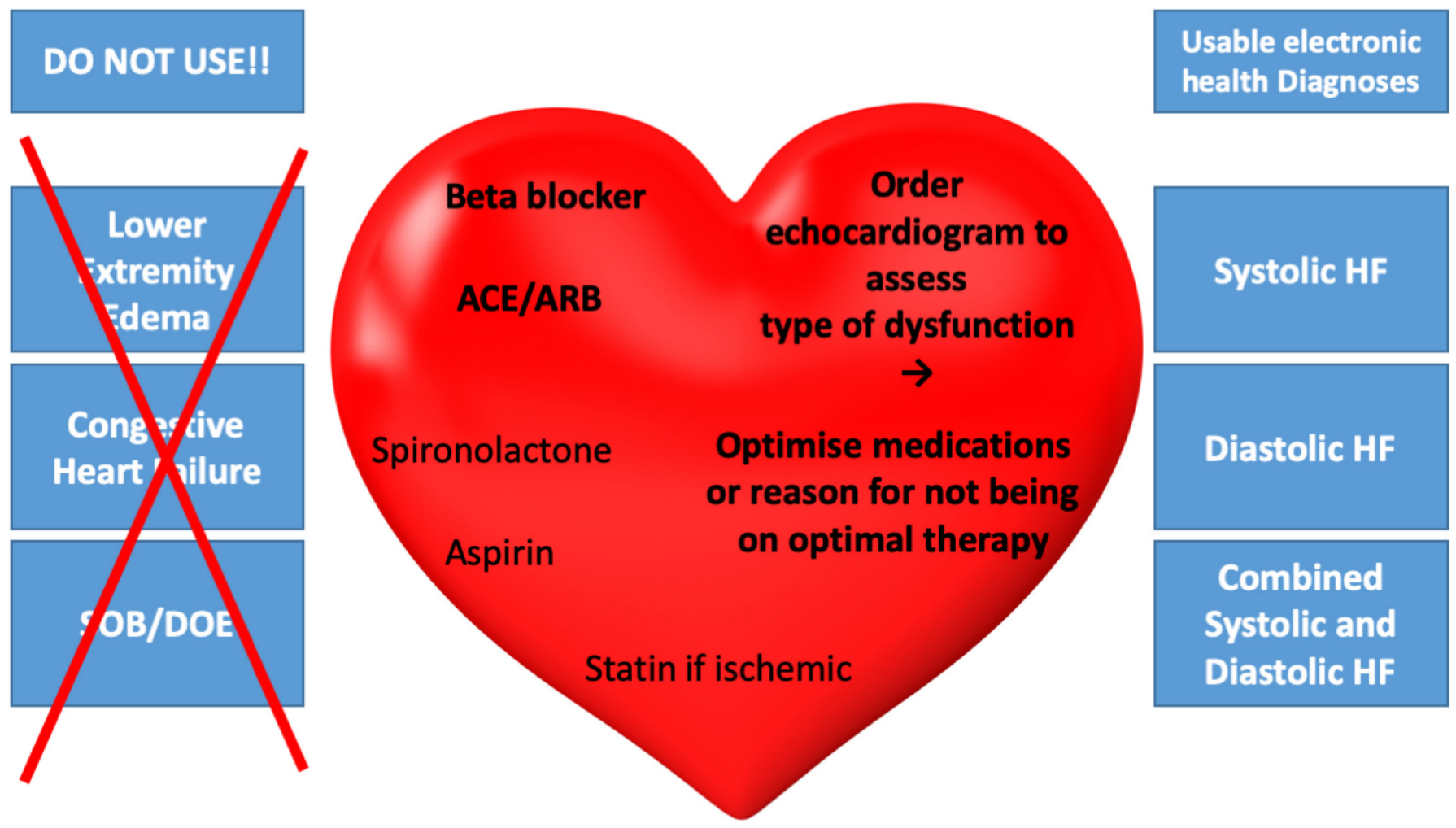

Figure 3b. Patient Education Pamphlets
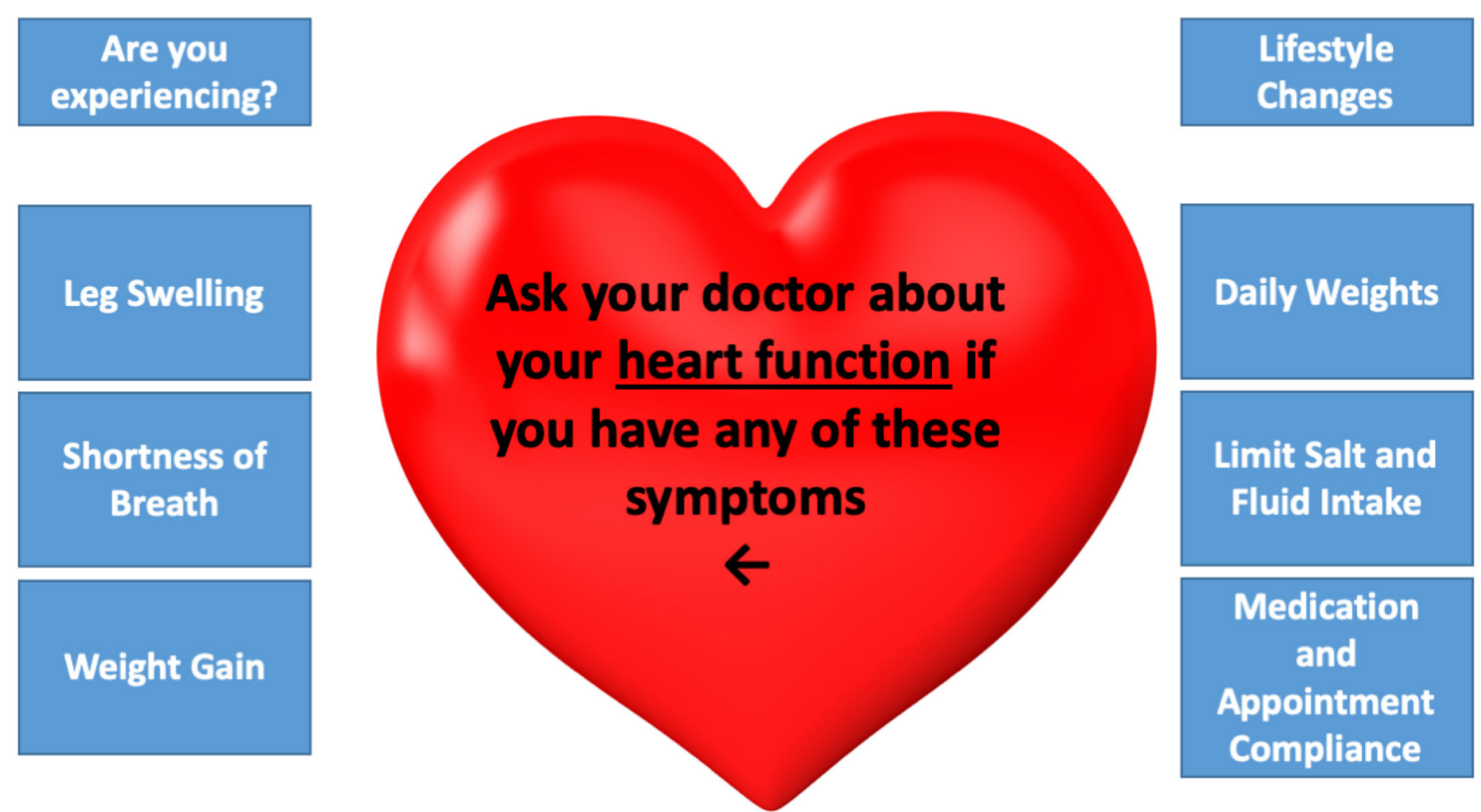

Figure 3 Reminder and educational pamphlets: (A) physician reminder pamphlets and (B) patient education pamphlets. ARB, angiotensin receptor blocker; HF, heart failure. 
A weekly Echocardiogram order rate

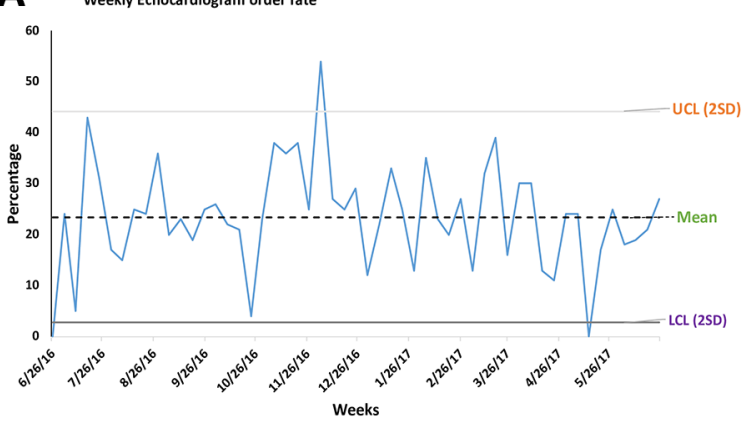

C Monthly Rates of Accurate Classification HF

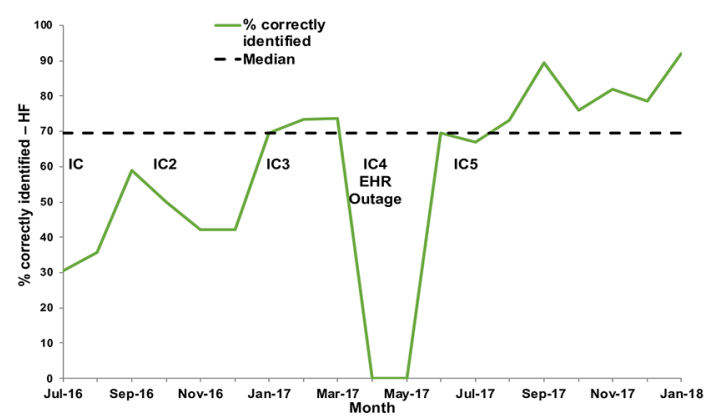

B

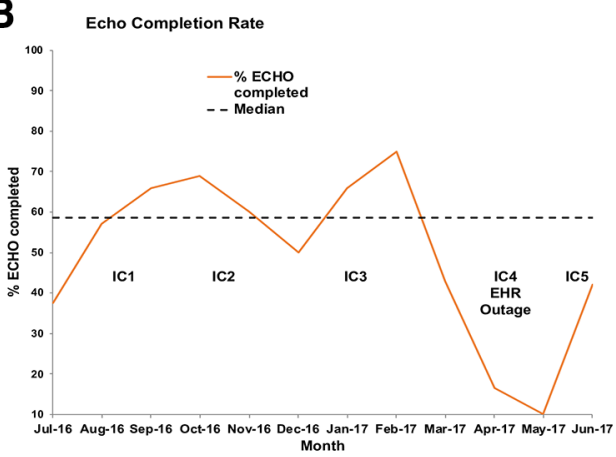

D

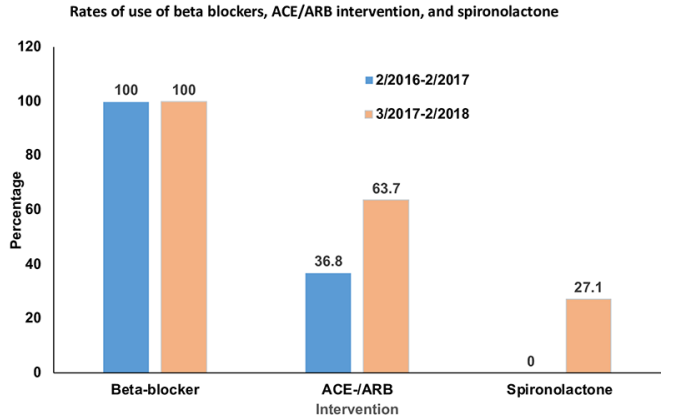

Figure 4 (A) Weekly statistical process control chart showing the percentage of the echocardiogram order rates. (B) Monthly run chart showing the percentage of the echocardiogram completion rates. (C) Monthly run chart showing the percentage of the $\mathrm{HF}$ classifications specified. (D) Bar chart showing the rates of use of ACE or ARBs, spironolactone. ARB, angiotensin receptor blocker; EHR, electronic health record; IC, improvement cycle.

calculated the total number of emergency department, hospitalisation and observation visits in the HF study population during the preintervention and postintervention intervals and compared the percentage of patients without any visit of each type during each interval using $\chi^{2}$ analyses. The same patients $(n=158)$ were included in both the preintervention and postintervention intervals, and since each preinterval and postinterval were for a period of 12 months, we found it acceptable to compare the total number of each type of visit during each interval.

\section{RESULTS}

\section{Demographics}

We analysed patient demographics in the clinic from July 2016 to June 2017. The majority of the 158 patients included in the project were African-American (72.8\%), with the remainder of the patients being white $(20.3 \%)$ or other races $(7.0 \%)$. The mean age was 64.6 years $(\mathrm{SD}=11.6)$, and most of the patients were male $(57.0 \%)$.

\section{Process measures}

\section{Echocardiogram order rate}

The mean of the weekly echocardiogram order rate in the SPC chart was $23.47 \%$. There was significant variation during various weeks (figure $4 \mathrm{~A}$ ).

\section{Echocardiogram completion rate}

The median monthly echocardiogram completion rate was $58.5 \%$ (figure 4B) within 12 months of this QI project. We achieved a $75.0 \%$ echocardiogram completion rate in February 2017, with variable rates during the other months. When calculating the median in the run chart, we did not include data for April and May 2017 due to the EHR outage.

\section{HF classification}

We achieved a sustainable increase in HF classification during the 12 months of this QI project (July 2016-June 2017). We also sustained a steady increase during the postproject period (July 2017-January 2018). The median during the project and postproject period from July 2016 to January 2018 was $70.0 \%$ (figure 4C). The accuracy of an HF diagnosis is dependent on an echocardiogram. During the QI project period, physicians reviewed echocardiograms that were completed within the last 2 years and corrected specific diagnosis of HF based on International Classification Diagnosis Codes. Physicians ordered a new echocardiogram if patients did not have an echocardiogram completed within the last 2 years. Therefore, the echocardiogram completion rate was lower than the rate of accurate HF classification. Physicians identified a gap in the accurate diagnosis of HF despite the completion of an echocardiogram within the last 2 years.

\section{Outcome measures}

Guideline-directed medical therapy

An increase in the use of ACE or ARBs from the baseline rates of $20 \%-37 \%$ occurred during the second half of the project. We achieved a sustainable increase, $71.4 \%$ median, during the postproject period of July 
2017-January 2018. We also increased the use of spironolactone from a baseline of $0 \%$ to a median of $27 \%$ in patients with systolic HF during the postproject period. All patients with systolic HF were on beta blockers during the preproject period and continued to maintain $100 \%$ usage during the project and the postproject period (figure 4D).

\section{Emergency department and hospital admissions}

In comparing 1-year preproject and 1-year postproject intervals, we observed reductions in the total number of emergency department visits (160 vs 108), hospital admissions (117 vs 114) and observation visits (22 vs 16) in the study population. As these data simply represent the total number (ie, count) of each type of visit during the preintervention and postintervention intervals, we did not perform any type of formal comparative hypothesis testing. We simply reported the absolute number of visits as formally testing the differences between means or proportions did not seem appropriate. Furthermore, there was an increase in the percentage of patients with no emergency department visits (53.8\% vs $63.3 \%)$ and observation visits $(88.6 \%$ vs $91.8 \%$ ), although these increases failed to reach statistical significance $(\mathrm{p}=0.087$ and 0.344 , respectively). A non-significant decrease in the percentage of patients with no hospitalisation visits $(68.4 \%$ vs $63.3 \%$, $\mathrm{p}=0.343$ ) was also observed.

\section{Balance measures}

\section{Increase in wait time}

The extra time spent to discuss echocardiogram results and shared decision for guideline-directed medical therapy with patients did not result in any significant increase in patient wait times in the clinic. There were no reports of an increase in patient complaints or overtime for clinic staff, which suggest that the project may not have adversely impacted patient wait times. Patients spent a total of 1 hour during a follow-up clinic visit, including wait and time with the provider. The average time spent in the clinic during a follow-up visit was about 1 hour prior to this QI. The time did not change during the project.

\section{Increase in wait time for echocardiogram}

Due to an increase in demand for echocardiogram and limited access, there was a delay in scheduling appointments. The wait time for echocardiogram before the project was about 1 month, and during the project period, it increased to about 2 months.

\section{Access to medications}

Physicians prescribed generic medications for our patients; therefore, the cost of medications was not a major barrier. A social worker facilitated access to medications in patients without health insurance or lack of transportation to obtain prescriptions.

\section{DISCUSSION}

This study describes the important topic of process improvement in the diagnosis and treatment of patients with HF. The study included multiple improvement cycles that address (1) leveraging health information technology; (2) optimising teamwork; and (3) providing education to physicians, staff and patients. This study was conducted in a tertiary safety-net hospital with residents coleading the improvement work, which makes the results particularly relevant to many healthcare providers and teams trying to improve in Hospital Readmission Reduction Programme and Delivery System Reform Incentive Payment programme initiatives. ${ }^{10-12}$ Clinical outcomes for patients with HF have been shown to be better when a patient is treated by a cardiologist, suggesting the need for education of general internists. ${ }^{20}{ }^{21}$ However, physician education on HF guideline-directed medical therapy has yielded conflicting results. Studies have shown improvements in appropriate prescribing and decreases in HF-related readmissions. ${ }^{22-24}$ Pocket cards with HF evidence-based interventions and structured educational programmes ${ }^{22}$ have been shown to be useful. Other studies have shown that educational programmes directed to family medicine and internal medicine physicians show no differences in prescribing patterns for HF management or improvement of patients' quality of life. ${ }^{20}{ }^{25}$ Physician education was one of the major interventions in this QI. However, we did not measure the improvement in physicians' knowledge by conducting pretests and post-tests before and after the education session; therefore, we cannot directly attribute our improvements to physician education. Engagement of patients and their families in HF education and self-management support has also been proven successful in reducing readmissions. ${ }^{26}$ Multiple studies have shown that providing patients with tools to manage their disease can have a direct effect on hospital admissions. ${ }^{27} 28$ This QI involved active engagement of patients with physicians and nursing staff. Patients received faceto-face education on HF and fluid management. Patients were also provided with tools, including pamphlets and at home weight monitoring scales. These interventions were most likely the reason for our QI success.

\section{Lessons learned}

The lack of a clinical decision support tool built in the EHR was identified as the biggest barrier. During the initial stage of this QI, physicians ordered an echocardiogram without fully explaining and discussing the reason with the patients, so the patients did not keep scheduled echocardiogram appointments. During subsequent follow-up visits, physicians rectified this error and started explaining the reason for the echocardiogram to patients prior to ordering it. This process led to improvement in adherence to scheduled echocardiogram appointments. Previsit planning by the nursing staff to identify patients due or overdue for an echocardiogram and the creation of a chart alert in EHR were not successful due to time constraints and limited resources. The team agreed to 
change this workflow and instructed physicians to identify eligible patients for echocardiogram. Furthermore, physicians reviewed and discussed guideline-directed medical therapy with patients. Engagement of a multidisciplinary team and emphasis on education to physicians, patients and staff were the key factors for the success of this project. Creation of a fishbone diagram to perform root cause analysis for suboptimal guideline-directed medical therapy for patients with $\mathrm{HF}$ and designing interventions to overcome the identified barriers were fundamental pillars for the design of this QI. The team meetings with internal medicine clinic residents, physicians and interdisciplinary teams resulted in positive feedback, which led to redesigning different interventions. We were unable to implement same-day (at the time of clinic visits) echocardiograms during the project period due to lack of resources and a need for prior authorisation from certain insurance (third-party payers); therefore, we scheduled echocardiogram appointment at a later date. We had multiple challenges to design the workflow to track no-shows for echocardiogram appointments and notification to the physician. Due to limited resources, we were unable to appoint a patient navigator or medical office assistant to track echocardiogram orders and completion rates and urgent referrals to cardiology.

\section{Limitations}

This QI was performed in a safety-net primary care clinic in patients with multiple comorbidities, so the barriers and interventions identified in this QI may not be generalisable to other settings. Due to limited resources in the clinic, we did not measure actual wait times during a clinic visit and wait times for scheduling an echocardiogram appointment during this QI project. Information technology was unable to generate these data from the electronic health database. We were unable to create a report to identify metrics specifically for hospital admissions versus readmissions for $\mathrm{HF}$ as the primary diagnosis; therefore, we analysed data for emergency department visits, hospital admissions and observations due to any medical reasons not specific to HF during the preproject and postproject periods. Results should be interpreted with caution, and the observed reduction in hospitalisation may not be directly attributable to the study interventions. Additionally, we did not measure the improvement in physicians' knowledge by conducting pretests and posttests; therefore, we could not attribute education to the improvement in our results.

\section{Sustainability and spread}

The hospital leadership has developed a culture supportive of QI. In the internal medicine clinic, the processes and workflows designed during this QI have become the standard of care for improving guideline-directed medical therapy for HF. We sustained an increase in accurate classification of $\mathrm{HF}$ at $80 \%$ during the postproject period (July 2017-January 2018). Strategies that were implemented in this quality QI can be replicated in other settings to improve diagnosis and guideline-directed medical therapy for patients with HF. There was a minimal cost involved in implementing this QI project.

\section{CONCLUSIONS}

We exceeded our goal and achieved $80 \%$ accurate classification of HF based on ejection fraction and significantly increased the use of guideline-directed medical therapy. A team-based approach that engaged leadership, internal medicine clinic and information technology staff resulted in improvement in guideline-directed medical therapy for patients with HF in a safety-net clinic. Future directions will include strategies to promote guideline-directed medical therapy based on the 2017 updated HF guidelines $^{3}$ and outreach of patients with HF that were lost to follow-up in the internal medicine clinic for population health.

Acknowledgements We thank the staff from the internal medicine clinic and information technology.

Contributors SB: study design, data collection, data analysis and manuscript draft; AB: data collection; HP: data collection; SV: data collection; GDG: data analysis; JLR: data analysis and manuscript draft.

Funding Research reported in this publication was supported by 1R01Al129649 (NIAID) (JR) and the National Center for Advancing Translational Sciences of the National Institutes of Health under award number UL1TR001412 (JR). The content is solely the responsibility of the authors and does not necessarily represent the official views of the NIH.

Competing interests None declared.

Patient consent for publication Not required.

Ethics approval This study was approved by the human subjects institutional review board of the University at Buffalo and was exempt from patient consent. The work was deemed a quality improvement project and not a study on human subjects.

Provenance and peer review Not commissioned; externally peer reviewed.

Open access This is an open access article distributed in accordance with the Creative Commons Attribution Non Commercial (CC BY-NC 4.0) license, which permits others to distribute, remix, adapt, build upon this work non-commercially, and license their derivative works on different terms, provided the original work is properly cited, appropriate credit is given, any changes made indicated, and the use is non-commercial. See: http://creativecommons.org/licenses/by-nc/4.0/.

\section{REFERENCES}

1 CDC. Heart failure fact sheet, 2016. Available: https://www.cdc.gov/ dhdsp/data_statistics/fact_sheets/fs_heart_failure.htm

2 Yancy CW, Jessup M, Bozkurt B, et al. 2013 ACCF/AHA Guideline for the Management of Heart Failure: Executive Summary. J Am Coll Cardiol 2013;62:1495-539.

3 Yancy CW, Jessup M, Bozkurt B, et al. 2017 ACC/AHA/HFSA focused update of the 2013 ACCF/AHA guideline for the management of heart failure: a report of the American College of Cardiology/American heart association Task force on clinical practice guidelines and the heart failure Society of America. Circulation 2017;136:e137-61.

4 Ziaeian B, Fonarow GC. The prevention of hospital readmissions in heart failure. Prog Cardiovasc Dis 2016;58:379-85.

5 Bergethon KE, Ju C, DeVore AD, et al. Trends in 30-day readmission rates for patients hospitalized with heart failure: findings from the get with the Guidelines-Heart failure registry. Circ Heart Fail 2016;9.

6 Kato N, Kinugawa K, Seki S, et al. Quality of life as an independent predictor for cardiac events and death in patients with heart failure. Circ J 2011;75:1661-9.

7 Kato NP, Okada I, Imamura T, et al. Quality of life and influential factors in patients implanted with a left ventricular assist device. Circ J 2015;79:2186-92. 
8 Nieminen MS, Dickstein K, Fonseca C, et al. The patient perspective: quality of life in advanced heart failure with frequent hospitalisations. Int J Cardiol 2015;191:256-64.

9 Mcllvennan CK, Eapen ZJ, Allen LA. Hospital readmissions reduction program. Circulation 2015;131:1796-803.

10 CMS.GOV. Hospital readmissions reduction program (HRRP). Available: https://www.cms.gov/medicare/medicare-fee-for-servicepayment/acuteinpatientpps/readmissions-reduction-program.html

11 Felland L, Lipson D, Heeringa J. Examining New York's Delivery System Reform Incentive Payment Demonstration: Achievements at the Demonstration's Midpoint and Lessons for Other States. M50 Mathematica Policy Research 2018

12 NYSDOH. DSRIP overview, 2014. Available: https://www.health.ny. gov/health_care/medicaid/redesign/dsrip/overview.htm

13 Bjerke MB, Renger R. Being smart about writing smart objectives. Eval Program Plann 2017;61:125-7.

14 Crossing the quality chasm: a new health system for the 21st century, 2001. Available: http://www.nationalacademies.org/hmd/ / media/Files/Report\%20Files/2001/Crossing-the-Quality-Chasm/ Quality\%20Chasm\%202001\%20\%20report\%20brief.pdf

15 The six domains of health care quality. Available: https://www. ahrq.gov/professionals/quality-patient-safety/talkingquality/create/ sixdomains.html

16 The CAHPS ambulatory care improvement guide: practical strategies for improving patient experience. Available: https://www.ahrq.gov/ cahps/quality-improvement/improvement-guide/improvement-guide. htm

17 Quality Improvement, 2011. Available: https://www.hrsa.gov/sites/ default/files/quality/toolbox/508pdfs/qualityimprovement.pdf

18 The drive for healthcare quality and pay for performance. Available: http://bhmpc.com/2014/01/drive-healthcare-quality-payperformance/
19 McQuillan RF, Wong BM. The Squire guidelines: a scholarly approach to quality improvement. J Grad Med Educ 2016;8:771-2.

20 Park LG, Mahar D, Shaw RE, et al. The impact of a heart failure educational program for physicians varies based upon physician specialty. J Clin Med Res 2014;6:173-83.

21 Reis SE, Holubkov R, Edmundowicz D, et al. Treatment of patients admitted to the hospital with congestive heart failure: specialtyrelated disparities in practice patterns and outcomes. J Am Coll Cardiol 1997;30:733-8.

22 Chua CC, Hutchinson A, Tacey M, et al. A physician targeted intervention improves prescribing in chronic heart failure in general medical units. BMC Health Serv Res 2018;18:206.

23 Miller RF. Management of heart failure in a rural community. Home Healthcare Now 2017;35:420-6.

24 Miller WL, Skouri HN. Chronic systolic heart failure, GuidelineDirected medical therapy, and systemic Hypotension-Less pressure but maybe more risk (does this clinical scenario need more discussion?). J Card Fail 2009;15:101-7.

25 Vaillant-Roussel H, Laporte C, Pereira B, et al. Impact of patient education on chronic heart failure in primary care (etic): a cluster randomised trial. BMC Fam Pract 2016;17:80.

26 D-I B, Mark H, Groff-Paris L, et al. A nurse-guided patient-centered heart failure education program. J Nurs Edu Pract 2014;4:1-9.

27 Simpson M. A quality improvement plan to reduce 30 -day readmissions of heart failure patients. J Nurs Care Qual 2014;29:280-6.

28 Boyde M, Peters R, Hwang R, et al. The self-care educational intervention for patients with heart failure. $J$ Cardiovasc Nurs 2017;32:165-70. 